\title{
Severe Vulvar and Perianal Crohn's Disease: A Case Report and Review of the Literature
}

\author{
Guillaume Le Cosquer $^{a} \quad$ Alexis Barranca ${ }^{b}$ Louise Lapotre ${ }^{b}$ \\ Eline Casassa ${ }^{b}$ Benoit Chaput ${ }^{c}$ Jonathan Ciron ${ }^{d}$ Louis Buscail ${ }^{a}$ \\ Carle Paul $^{\text {b }}$ Cyrielle Gilletta ${ }^{a}$ \\ aDepartment of Gastroenterology, Toulouse University Hospital, Toulouse, France; \\ ${ }^{b}$ Department of Dermatology, Toulouse University Hospital, Toulouse, France; 'Department \\ of Plastic, Reconstructive, Aesthetic and Burns Surgery, Toulouse University Hospital, \\ Toulouse, France; dDepartment of Neurology, Toulouse University Hospital, Toulouse, \\ France
}

\section{Keywords}

Pyoderma gangrenosum $\cdot$ Vulvar Crohn's disease $\cdot$ Cyclosporin $\cdot$ Infliximab

\begin{abstract}
Association of vulvar Crohn's disease (CD) with pyoderma gangrenosum (PG) has been described, yet due to its low prevalence, data on therapeutic management of this association are still limited. We here present a 22-year-old woman with severe vulvar and perianal CD with a major inguinal and perineal ulceration. In the hypothesis of an associated PG, oral corticosteroids were prescribed at a dose of $40 \mathrm{mg}$ with limited efficacy for a duration of 3 months. Afterward, a combination treatment of cyclosporine and infliximab followed by negative pressure wound therapy and split-thickness skin graft resulted in complete healing.
\end{abstract}

\section{Introduction}

Cutaneous manifestations of Crohn's disease (CD) may be classified into three categories (excluding treatment induced manifestations): specific, reactive, and associated [1]. Specific manifestations are noncaseating granulomatous skin lesions separated from the affected 
gastrointestinal tract by healthy tissue, also known as metastatic CD. Vulvar CD (VCD) is a rare specific manifestation with less than 200 cases reported [2]. It is associated with intestinal lesions or perianal lesions in $80 \%$ and about $50 \%$ of cases, respectively. It may also precede the digestive manifestations of $\mathrm{CD}$ in 1 case out of 4 [2].

Reactive manifestations share common pathophysiology, such as pyoderma gangrenosum (PG) and Sweet's syndrome. PG is an autoinflammatory neutrophilic dermatosis with complex and multifactorial pathophysiology that involves neutrophilic dysfunction, inflammatory mediators, and genetic predisposition [3]. The prevalence of PG in inflammatory bowel disease (IBD) ranges from 0.4 to $2 \%$ [4]. IBD is the first associated condition with PG accounting for up to $30 \%$ of cases. PG can take several forms, the most frequent being single or multiple painful erythematous papules or pustules typically occurring on the lower legs. The lesions evolve into ulcerations, containing sterile purulent material [3]. Its diagnosis is clinical and must be retained after the exclusion of other skin diseases (infectious, vascular, and other autoimmune conditions). Finally, associated skin lesions are often associated with CD but without associated pathogenic mechanism (e.g., erythema nodosum). We here report a rare case of VCD associated with a suspected lesion of PG.

\section{Case Report/Case Presentation}

A 22-year-old patient presented to the Emergency Department of our hospital with an abscessed lesion of the left buttock and the perineum. Patient's symptoms started 2 weeks ago with the appearance of an abscessed lesion of the left buttock, which had been worsened the last $48 \mathrm{~h}$. The patient was diagnosed with VCD at the age of 21 years based on vulvar edema (shown in Fig. 1), anal pain and anal ulceration at colonoscopy, granulomas on colonic biopsies, and eventually an elevated fecal calprotectin level.

Her medical history begins at the age of 15 years with a severe Devic's syndrome, which is a neuromyelitis characterized by antibodies against aquaporin 4 . This pathology was revealed 6 years ago by tetraplegia, facial diplegia, and respiratory distress initially requiring intensive care and initiation of rituximab, which was pursued for 2 years. The etiology selected was paraneoplastic, due to a left ovarian teratoma subsequently resected.

The following year, she suffered from diarrhea while she was treated by rituximab requiring investigation by a colonoscopy. This exam showed a pancolitis with loss of vascularity and aphthous ulcers. Analysis of the random colonic biopsies revealed active inflammation leading at this time to the diagnosis of infectious colitis, well treated with antibiotics. In these biopsies, few granulomas were found which allows to retrospectively establish the diagnosis of $\mathrm{CD}$.

Fig. 1. Picture of the initial clinical presentation as a vulvar edema.

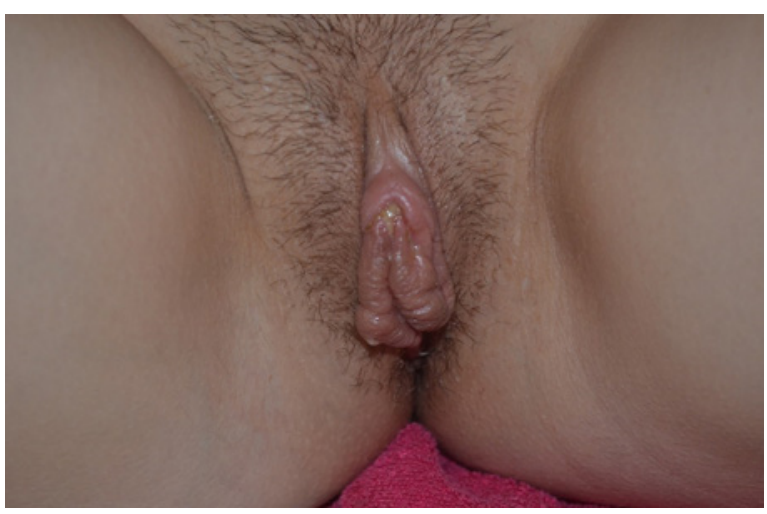




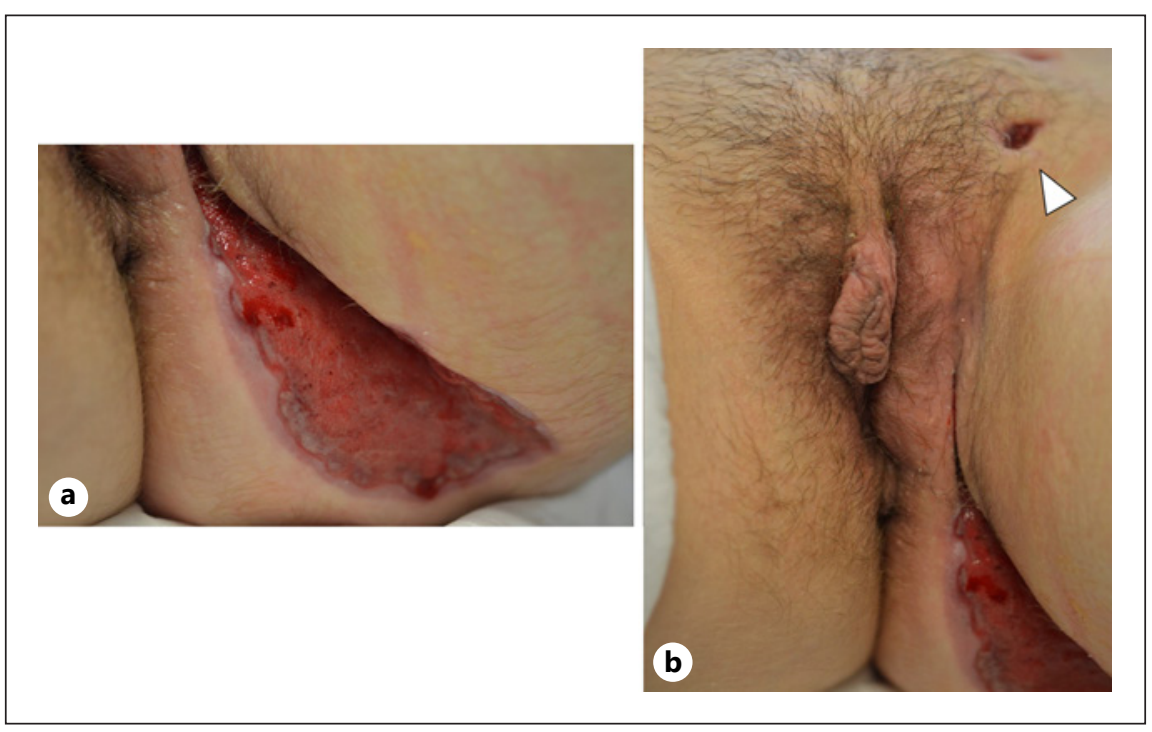

Fig. 2. Picture of the pelvis suspected PG with para-vulvar fistula. a Suspected PG measured at $10 \mathrm{~cm}$ from the major axis after excision and with inflammatory borders of the left buttock. $\mathbf{b}$ Lower magnification of the suspected PG, inflammation of the left buttock, and ano-vulvar fistula (with arrowhead).

From the age of 17 to 21 years, she suffered from vulvar edema and erosions and had received various treatments with several antibiotics and colchicine without success. At last, a colonoscopy was performed, motivated by anal pain and a raised calprotectin level. An ulceration of the anal canal was found, which has been biopsied. The histological analysis of the biopsies was nonspecific. No trace of active disease was found on the colon and terminal ileum during the exam. Finally, the diagnosis of VCD was made, after a delay due to this untypical presentation. Taking into account the medical history, TNF- $\alpha$ inhibitors were not considered adequate, and after a multidisciplinary discussion, ustekinumab (90 mg every 4 weeks after initiation) was started in combination with azathioprine $(2 \mathrm{mg} / \mathrm{kg} /$ day $)$.

One month after initiation, the patient was admitted to the emergency department for the lesion we herein report. Upon admission, the clinical dermatological examination revealed an abscessed lesion of the left buttock and the perineum. Away from the lesion, an external opening of a suspected ano-vulvar fistula was also noticed. The exact path of the fistula (from the anus to the vulva) was confirmed later on MRI. Due to progression of the inflammation despite systemic antibiotics, a surgical excision of the lesion was performed (shown in Fig. 2). All the bacteriological skin samples were negative, and the histological analysis found acanthotic and parakeratotic epidermis with a weakly ulcerated center containing polynuclear neutrophil infiltration extending to the hypodermis. The dermis contained an inflammatory infiltrate containing a predominance of mononuclear cells with perivascular and periadnexal tropism.

The diagnosis of PG associated with VCD was suspected on the dermatological aspect, the negative bacteriological culture, and the exclusion of other skin diseases on the histological analysis. The main differential diagnosis was a manifestation of her perineal CD. Oral corticosteroids were prescribed at a dose of $40 \mathrm{mg}$ with limited efficacy for a duration of 3 months. After multidisciplinary expert consultation, it was decided to initiate a combination therapy with infliximab and cyclosporine.

This therapeutic option was debated and approved during a national neurological consultation meeting. Indeed, the etiology of Devic's syndrome being paraneoplastic (with resected tumor), there was no flare-up of the disease since the diagnosis, allowing the patient to 


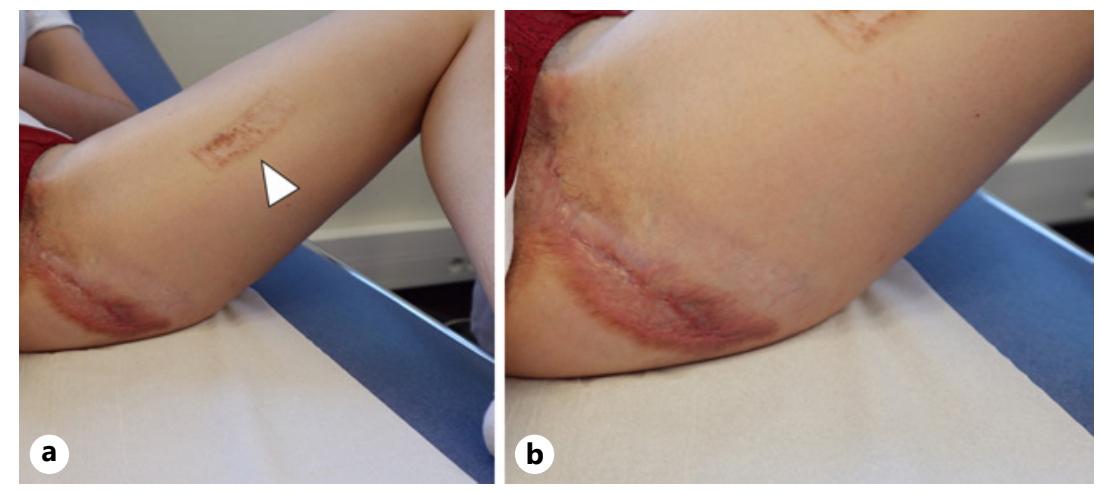

Fig. 3. Healing of the lesions after reconstructive surgery and combination of biotherapy. a Healing after skin graft (rectangular scar of the donor site on the left thigh; white arrowhead) and combination therapy of infliximab and cyclosporine. b Zoom on the healing lesion.

be considered in remission. Furthermore, there is no reported case of a flare-up of Devic's syndrome under TNF alpha inhibitors reported in the literature (contrary to multiple sclerosis). A close follow-up must be planned, with regular clinical examination, neurological MRI, and biological monitoring of the antibodies against aquaporin 4. Therefore, a combination therapy with infliximab (5 mg/kg every 4 weeks after standard induction: week $0,4,8)$ and cyclosporine ( $4 \mathrm{mg} / \mathrm{kg} /$ day) was initiated while tapering oral corticosteroids.

After 3 months of combination therapy with infliximab and cyclosporine, concomitant negative pressure (vacuum-associated closure) was applied to the skin ulceration to promote healing. The next month, complete clearance of local inflammation was obtained, and an autologous split-thickness skin graft was performed, followed by 4 days of negative pressure therapy on the surgical site.

Three months after the surgery, the patient was in clinical and biological remission of CD, with a Harvey-Bradshaw index decreasing from 7 initially to 2 and fecal calprotectin levels decreasing from 797 to $103 \mu \mathrm{g} / \mathrm{g}$ of stools. Therapeutic de-escalation was decided on, first by lowering the dose of cyclosporine $(2 \mathrm{mg} / \mathrm{kg} /$ day) 2 months after the skin graft. Then, cyclosporine was replaced by azathioprine $(2 \mathrm{mg} / \mathrm{kg} /$ day $)$ in the 6 th month. Despite that, the combination therapy with infliximab and cyclosporine was well tolerated, and we choose to replace cyclosporine by azathioprine because of the potential severe adverse events in long term. This strategy allowed a complete healing of the skin's lesions at 6 months (shown in Fig. 3).

\section{Discussion}

The association of VCD and PG has been described in up to 20\% of VCD cases in some series [2]. The originality of our case resides in our therapeutic choice with the combination therapy of infliximab and cyclosporine.

Owing of the lack of data on VCD, no consensus on diagnostic or therapeutic management has been published. The issue of diagnosis must be split into two situations. First, in the case of diagnosed CD patients who develop vulvar lesions afterward (the diagnosis of CD), the key part for the gastroenterologist is to exclude lesions due to fistulas from the affected bowel and then to refer the case to an expert dermatologist to ensure the diagnosis. Patients whose first presentation of $\mathrm{CD}$ is vulvar are tougher to diagnose; Boxhoorn et al. [5] reported a median diagnosis delay of 3 years and 6 months. In those cases, patients are initially referred to dermatologists and gynecologists. The diagnosis of CD must be suspected as soon as other 
skin disease has been excluded (especially infectious and granulomatous diseases), and skin biopsy shows the presence of noncaseating granulomas, which has been reported in $80 \%$ of cases [6].

In the largest available prospective study (regrouping 31 cases), Bhoyrul and Lyon [2] suggest adopting a multidisciplinary approach (dermatologists, gastroenterologists, and gynecologists) to take care of VCD. Topical corticosteroids (or tacrolimus) are effective in mild VCD, defined as limited erosions and superficial fissures. Metronidazole seems to be an option alone or in combination with topicals [7]. The second-line therapy (or first-line for severe VCD) relies on topical therapy in combination with immunosuppressants. TNF alpha inhibitors appear to be the most effective immunosuppressants with about 70\% complete healing [6].

There is a poor evidence base for management of $\mathrm{PG}$, with only two randomized control trials. Ormerod and al show no difference between prednisolone and cyclosporine, with about $50 \%$ healing at 6 months [8]. Yet, more serious adverse reactions were reported in the steroid group (serious infections) than in the cyclosporine group (renal toxicity). Brooklyn et al. [9] demonstrate the superiority of infliximab over a placebo with a clinical response in about $69 \%$ of patients. A step-up approach is the most widespread, with corticosteroids or cyclosporine as first-line therapy, followed by immunosuppressives or biologics in severe cases [10].

The last European evidence-based consensus on extra intestinal manifestations in IBD recommends treating PG with systemic corticosteroids, an anti-TNF agent or a topical (or oral) calcineurin inhibitor [11]. Although, a recent systematic review shows that an anti-TNF agent and cyclosporine demonstrated efficacy for healing PG, whereas steroids seemed to perform poorly, unless started early at high doses, in cases of associated CD [12].

PG is associated with a pathergy phenomenon in $30 \%$ of cases, which is characterized by progression or emergence of lesions after trauma or surgery [13]. Thus, the place of surgery in the therapeutic approach of PG is controversial. However, case series of successful skin grafts in PG associated with IBD have been reported [14]. A recent systematic review of surgical treatment of PG reports 73\% success of split-thickness skin grafts, alone or followed by negative-pressure wound therapy, under concomitant successful immunosuppressive therapy [15].

\section{Conclusion}

We report for the first time the use of combination of infliximab, the cornerstone of CD treatment, cyclosporine, and split-thickness skin graft followed by negative-pressure wound therapy to manage a suspected PG associated with VCD. This combination constitutes an alternative option to oral corticosteroids with no impairment of the skin healing process.

\section{Statement of Ethics}

Ethical approval was not required for this report in accordance with national guidelines. Written informed consent was obtained from the patient for publication of this report and any accompanying images.

\section{Conflict of Interest Statement}

G.L.C.: none; A.B.: none; L.L.: none; E.C.: none; B.C.: none; J.C.: personal fees from Biogen, Novartis, Roche, Celgene-BMS, Merck, and Genzyme-Sanofi, all outside the submitted work; L.B.: none; C.P.: personal fees from Almirall, Amgen, Pfizer, Leo Pharma Regeneron, and Merck 
and grants and personal fees from AbbVie, Boehringer, Celgene, Eli Lilly, Janssen, UCB, Pierre Fabre, and Sanofi, all outside the submitted work; and C.G.: personal fees from Takeda, Pfizer, Janssen, AbbVie, Celltrion, Sandoz, and Mylan, all outside the submitted work.

\section{Funding Sources}

The authors received no financial support for the research, authorship, and publication of this article.

\section{Author Contributions}

Drs. Guillaume Le Cosquer, Alexis Barranca, and Louise Lapotre contributed to conception, design, interpretation, and manuscript preparation. Drs. Eline Casassa, Jonathan Ciron, Cyrielle Gilletta, Prs Benoit Chaput, Louis Buscail, and Carle Paul contributed to acquisition, analysis, and interpretation of data for the work and critically revising of the manuscript for important intellectual content. All the authors read and approved the final version manuscript.

\section{Data Availability Statement}

All data generated or analyzed during this study are included in this. Further inquiries can be directed to the corresponding author.

\section{References}

1 Greuter T, Navarini A, Vavricka SR. Skin manifestations of inflammatory bowel disease. Clin Rev Allergy Immunol. 2017 Dec;53(3):413-27.

2 Bhoyrul B, Lyon C. Crohn's disease of the vulva: a prospective study. J Gastroenterol Hepatol. 2018 Dec;33(12): 1969-74.

3 Alavi A, French LE, Davis MD, Brassard A, Kirsner RS. Pyoderma gangrenosum: an update on pathophysiology, diagnosis and treatment. Am J Clin Dermatol. 2017 Jun;18(3):355-72.

4 Vavricka SR, Schoepfer A, Scharl M, Lakatos PL, Navarini A, Rogler G. Extraintestinal manifestations of inflammatory bowel disease. Inflamm Bowel Dis. 2015 Aug;21(8):1982-92.

5 Boxhoorn L, Stoof TJ, de Meij T, Hoentjen F, Oldenburg B, Bouma G, et al. Clinical experience and diagnostic algorithm of vulval Crohn's disease. Eur J Gastroenterol Hepatol. 2017;29(7):838-43.

6 Laftah Z, Bailey C, Zaheri S, Setterfield J, Fuller LC, Lewis F. Vulval Crohn's disease: a clinical study of 22 patients. J Crohns Colitis. 2015 Apr;9(4):318-25.

7 Andreani SM, Ratnasingham K, Dang HH, Gravante G, Giordano P. Crohn's disease of the vulva. Int J Surg. 2010; 8(1):2-5.

8 Ormerod AD, Thomas KS, Craig FE, Mitchell E, Greenlaw N, Norrie J, et al. Comparison of the two most commonly used treatments for pyoderma gangrenosum: results of the STOP GAP randomised controlled trial. BMJ. 2015 Jun;350:h2958.

9 Brooklyn TN, Dunnill MGS, Shetty A, Bowden JJ, Williams JDL, Griffiths CEM, et al. Infliximab for the treatment of pyoderma gangrenosum: a randomised, double blind, placebo controlled trial. Gut. 2006 Apr;55(4):505-9.

10 Ahn C, Negus D, Huang W. Pyoderma gangrenosum: a review of pathogenesis and treatment. Expert Rev Clin Immunol. 2018 Mar;14(3):225-33.

11 Harbord M, Annese V, Vavricka SR, Allez M, Barreiro-de Acosta M, Boberg KM, et al. The first European evidence-based consensus on extra-intestinal manifestations in inflammatory bowel disease. J Crohns Colitis. 2016;10(3):239-54.

12 Partridge ACR, Bai JW, Rosen CF, Walsh SR, Gulliver WP, Fleming P. Effectiveness of systemic treatments for pyoderma gangrenosum: a systematic review of observational studies and clinical trials. Br J Dermatol. 2018 Aug;179(2):290-5.

13 Braswell SF, Kostopoulos TC, Ortega-Loayza AG. Pathophysiology of pyoderma gangrenosum (PG): an updated review. J Am Acad Dermatol. 2015 Oct;73(4):691-8. 
14 Andrisani G, Guidi L, Papa A, Potenza AE, Cervelli D, Armuzzi A. A case of pyoderma gangrenosum with ulcerative colitis treated with combined approach: infliximab and surgery. J Crohns Colitis. 2013 Jun;7(5):421-6.

15 Pichler M, Thuile T, Gatscher B, Tappeiner L, Deluca J, Larcher L, et al. Systematic review of surgical treatment of pyoderma gangrenosum with negative pressure wound therapy or skin grafting. J Eur Acad Dermatol Venereol. 2017 Feb;31(2):e61-7. 